\title{
Whole-Genome Shotgun Sequencing
}

National Cancer Institute

\section{Source}

National Cancer Institute. Whole-Genome Shotgun Sequencing. NCI Thesaurus. Code C19653.

A DNA sequencing method, which involves random sequencing of tiny cloned pieces of the genome, with no foreknowledge of where on a chromosome the piece originally came from. The sequences obtained have a considerable overlap and by using appropriate computer software it is possible to compare sequences and align them to build larger units of genetic information. This sequencing strategy can be automated and leads to rapid sequencing information. 